\title{
Erratum to: Cortical reorganization in an astronaut's brain after long-duration spaceflight
}

\author{
Athena Demertzi ${ }^{2}$ - Angelique Van Ombergen ${ }^{1} \cdot$ Elena Tomilovskaya $^{3} \cdot$ \\ Ben Jeurissen $^{4}$ - Ekaterina Pechenkova ${ }^{5}$. Carol Di Perri ${ }^{2} \cdot$ Liudmila Litvinova $^{5}$. \\ Enrico Amico $^{2}$ - Alena Rumshiskaya ${ }^{5}$ Ilya Rukavishnikov ${ }^{3} \cdot$ Jan Sijbers $^{4}$. \\ Valentin Sinitsyn $^{5} \cdot$ Inessa B. Kozlovskaya $^{3} \cdot$ Stefan Sunaert $^{6} \cdot$ Paul M. Parizel $^{7}$. \\ Paul H. Van de Heyning ${ }^{1} \cdot$ S. Laureys ${ }^{2} \cdot$ Floris L. Wuyts $^{1}$
}

Published online: 15 April 2016

(C) Springer-Verlag Berlin Heidelberg 2016

\section{Erratum to: Brain Struct Funct DOI 10.1007/s00429-015-1054-3}

The original article was published with the following errors:

1. The order of the two first authors was incorrect. Athena Demertzi should be ordered first and Angelique Van
Ombergen should be ordered second. Athena Demertzi and Angelique Van Ombergen are co-first authors with equal contributions.

2. The author name "Steven S. L. Laureys" should be changed to "S. Laureys".

The errors have been corrected in the original article.

The online version of the original article can be found under doi:10.1007/s00429-015-1054-3.

Angelique Van Ombergen

angelique.vanombergen@uantwerpen.be

1 Antwerp University Research Centre for Equilibrium and Aerospace (AUREA), Antwerp University Hospital and University of Antwerp, Groenenborgerlaan 171, 2020 Antwerp, Belgium

2 Coma Science Group, Cyclotron Research Centre and Neurology Department, University of Liège, Liège, Belgium

3 SSC RF, Institute of Biomedical Problems, Russian Academy of Sciences, Moscow, Russia

4 iMinds/Vision Lab, University of Antwerp, Antwerp, Belgium

5 Radiology Department, Federal Center of Treatment and Rehabilitation, Moscow, Russia

6 Department of Imaging and Pathology, Translational MRI, KU Leuven, Leuven, Belgium

7 Radiology Department, Antwerp University Hospital and University of Antwerp, Antwerp, Belgium 